\title{
Protection to Tomato Wilt Disease Conferred by the Nonpathogen Fusarium oxysporum Fo47 is More Effective Than that Conferred by Avirulent Strains
}

\author{
Francisco J. de Lamo, Staf B. Spijkers, and Frank L. W. Takken ${ }^{\dagger}$ \\ Molecular Plant Pathology, Faculty of Science, Swammerdam Institute for Life Sciences, University of Amsterdam, Amsterdam, \\ the Netherlands \\ Accepted for publication 24 July 2020.
}

\begin{abstract}
Although the vascular pathogen Fusarium oxysporum is notorious for being the causal agent of Fusarium wilt disease, the vast majority of F. oxysporum strains are harmless soil and root colonizers. The latter F. oxysporum's are often endophytes colonizing roots intracellularly without negatively affecting plant fitness. Actually, most of them, like Fo47, are beneficial providing biological control to various root pathogens. Interestingly, also pathogenic $F$. oxysporum inoculated on a resistant host (i.e., avirulent $F$. oxysporum f. sp. lycopersici) can reduce susceptibility to virulent $F$. oxysporum strains via a mechanism called "cross protection." It has been hypothesized that cross protection is based on activation of a resistance protein of the host upon recognition of a cognate avirulence (Avr) protein of the pathogen. Currently, it is unknown whether the biocontrol activity of $F$. oxysporum endophytes utilizes
\end{abstract}

ABSTRACT similar mechanisms as cross protection conferred by avirulent pathogens, and whether both provide a quantitative similar level of protection. Here, we show that in tomato biocontrol activity of the Fo47 endophyte to the pathogen $F$. oxysporum f. sp. lycopersici is more effective than cross protection induced by avirulent $F$. oxysporum f. sp. lycopersici strains activating either I, I-2, or both resistance proteins upon recognition of Avr1 or the Avr2/Six 5 pair, respectively. These findings imply that cross protection and biological control utilize different mechanisms to reduce susceptibility of the host to subsequent infections.

Keywords: Fusarium oxysporum, biological control, cross protection, disease control and pest management, endophyte-mediated resistance, genetics and resistance, tomato
Fusarium oxysporum, a filamentous ascomycete, is the causal agent of Fusarium wilt disease (Michielse and Rep 2009). This soilborne vascular fungus ranks among the top 10 major fungal plant pathogens (Dean et al. 2012). Each F. oxysporum pathogen harbors a specific set of effector genes required for pathogenicity on a specific host (van Dam et al. 2016). Nevertheless, the ample majority of $F$. oxysporum strains are nonpathogenic saprotrophs able to endophytically colonize plant roots (Bao et al. 2004). It has been well established that wilt disease-suppressive soils contain beneficial $F$. oxysporum endophytes that are responsible for protecting a susceptible host to pathogenic $F$. oxysporum strains (Alabouvette 1986; Tamietti et al. 1993). These F. oxysporum endophytes exert biological control by directly affecting the invading pathogen and/or by inducing plant immune responses. The latter response, also referred to as endophyte-mediated resistance (EMR), results in induction of a systemic immune response halting a wide variety of root-infecting pathogens, but typically not shoot-infecting pathogens (de Lamo and Takken 2020). EMR appears distinct from the well-established systemic acquired resistance (SAR) and induced systemic resistance (ISR) responses as, at least in tomato (Solanum lycopersicum), it is independent of the defense hormones salicylic acid, jasmonate, and ethylene (Constantin et al. 2019). Furthermore, whereas EMR and ISR are typically induced by nonpathogenic root colonizers, SAR can be triggered in incompatible interactions in which an avirulent pathogen infects a resistant host. Infection by a pathogen producing

${ }^{\dagger}$ Corresponding author: F. L. W. Takken; F.L.W.Takken@uva.nl

Funding: Support was provided by Nederlandse Organisatie voor Wetenschappelijk Onderzoek (Earth and Life Sciences VICI project no. 865.14.003) and H2020 Marie Skłodowska-Curie Actions (No. 676480, International Training Network BestPass).

The author(s) declare no conflict of interest.

(C) 2021 The American Phytopathological Society an avirulence (Avr) protein that is perceived by a matching host resistance $(\mathrm{R})$ protein results in activation of effector triggered immunity (ETI) (Pieterse et al. 2014). Resistant plants infected with such an avirulent $F$. oxysporum pathogen show a reduction in disease symptoms upon co-, or subsequent, inoculation with a virulent $F$. oxysporum strain (Biles and Martyn 1989; HuertasGonzalez et al. 1999). The observed resistance response, called cross protection, has been found to be effective in reducing Fusarium wilt symptoms in crops such as watermelon co-inoculated with an avirulent and a virulent $F$. oxysporum f. sp. niveum (pathogen of watermelon) isolate (Biles and Martyn 1989). Likewise, co-inoculation of tomato with a virulent and an avirulent F. oxysporum f. sp. lycopersici race 1 (pathogen of tomato) isolate resulted in cross protection (Huertas-Gonzalez et al. 1999). In the latter study, cross protection was also observed in melon upon coinoculation with virulent and avirulent strains of $F$. oxysporum $\mathrm{f}$. sp. melonis (pathogen of melon) (Huertas-Gonzalez et al. 1999).

Whereas endophytic $F$. oxysporum strains trigger EMR, cross protection induced by avirulent $F$. oxysporum strains could be a combination of EMR and ETI-triggered SAR, or only involve the latter. To study whether EMR- and cross protection-signaling pathways interact and affect each other, we compared cross protection induced by avirulent $F$. oxysporum pathogens with biological control exerted by $F$. oxysporum endophytes. Thereto, we used tomato cultivar Motelle carrying the $R$ genes $I$ and $I-2$ that confer resistance to $F$. oxysporum f. sp. lycopersici races 1 and 2 (Laterrot 1993; Takken and Rep 2010). I encodes a receptor-like protein (RLP) detecting the presence of extracellular $F$. oxysporum f. sp. lycopersici Avr1 (Catanzariti et al. 2017), while I-2 is a nucleotide-binding leucine-rich-repeat-type receptor (NLR) detecting intracellular Avr2 that requires Six 5 for its translocation through the host symplast and perception by I-2 (Cao et al. 2018; Houterman et al. 2009; Ma et al. 2015; Simons et al. 1998). A F. oxysporum f. sp. lycopersici isolate carrying only Avr2 or Six 5 is therefore virulent on $I-2$ containing tomato varieties (Ma et al. 2015). The use of the Motelle cultivar allows us to quantify and compare the 
level of wilt disease resistance induced by either endophytic $F$. oxysporum (=EMR) or avirulent $F$. oxysporum f. sp. lycopersici strains triggering either $I$ - or $I$-2-mediated immunity (=ETItriggered SAR and maybe EMR).

By using various host-pathogen combinations, we examined whether cross protection triggered by the RLP I is equivalent to, or different from, that activated by the NLR I-2. The following four avirulent $F$. oxysporum f. sp. lycopersici strains were used to activate I and/or I-2: Fol004 (race 1 carrying Avrl and Avr2/Six5), Fol004 $\Delta$ Six 5 (race 1 carries Avrl and Avr2 but lacks Six5), Fol004 $\triangle A v r 1$ (race 2 carries Avr2/Six 5 but not Avr1), and Fol007 (race 2 carrying Avr2/Six 5 but not Avrl) (Table 1). As virulent control Fol029 (race 3) lacks Avr1 and carries a single amino acidic substitution in the Avr2 protein allowing it to evade I and I-2 mediated recognition (Houterman et al. 2009). By using tomato cultivar Motelle (carrying $I$ and $I-2$ ), these strains allow us to study whether cross protection against the virulent race 3 Fol029 isolate triggered by the I cell surface immune receptor is quantitatively different from that triggered by the intracellular I-2 receptor.

As expected, bipartite interactions of tomato cultivar Motelle with either avirulent $F$. oxysporum f. sp. lycopersici pathogens or with Fo47 (Fig. 1A, upper row panels) did not result in disease symptoms, such as a reduction of plant fresh weight $(\mathrm{FW})$ compared with the mock (water-inoculated) control (Fig. 1B). In contrast, and as anticipated, inoculation with the virulent Fol029 (race 3) strain resulted in severe disease symptoms such as stunting and wilting (Fig. 1A, bottom left panel), concomitant with a strongly reduced FW of the infected plants (Fig. 1B). The disease index (DI) of tomato inoculated with Fol029 differed significantly from seedlings inoculated with either of the four avirulent $F$. oxysporum f. sp. lycopersici pathogens or Fo47 (Fig. 1C). These results are as predicted based on the combination of plant and fungal genotypes employed; no disease symptoms occurred when plants were inoculated with avirulent $F$. oxysporum f. sp. lycopersici strains while the virulent strain caused disease.

The virulent Fol029 strain was subsequently co-inoculated with each of the four avirulent $F$. oxysporum f. sp. lycopersici strains separately to assess their potential to induce cross protection and reduce disease symptoms caused by the pathogen. When all treatments are compared using a Kruskal-Wallis test (Dunn's multiple comparison test), then plant weight following all inoculation involving Fol029 differed significantly $(P<0.0001)$ from the mock, except when Fol029 was co-inoculated with Fo47 (47: $029)$. The nonsignificant $P$ value $(P=0.06)$ for the latter pair shows that Fo47 confers protection against the pathogenic Fol029 strain as plant weight did not significantly differ from the mock. Notably, in single comparisons (using a Mann-Whitney test), cross protection was triggered in tomato by each of the avirulent strains, as coinoculation with Fol029 led to a significant higher FW (Fig. 1B) and a lower DI (Fig. 1C) than tomato plants infected solely with Fol029.
No differences in the extent of cross protection were found when Fol029 was co-inoculated with any of the avirulent $F$. oxysporum f. sp. lycopersici strains that activate either I or I-2 (Fig. 1) as no significant differences were found in FW and DI (Fig. 1B and C, compare treatments 004:029, 004 $\Delta$ Six 5:029, 004 $\Delta A v r 1: 029$, and 007:029-). As $I$ has been reported to restrict host colonization by race $1 F$. oxysporum $\mathrm{f}$. sp. lycopersici strains to a higher extent than I-2 halting race 2 isolates (van der Does et al. 2018), one would anticipate $I$ to confer a stronger containment toward Fol029 than $I-2$. Indeed, some tomato plants inoculated with either Fol004 $\triangle A v r 1$ or Fol007 (both races 2 only triggering I-2) exhibited brown vessels at stem cross sections at the height of the cotyledons indicating the presence of $F$. oxysporum f. sp. lycopersici (i.e., DI 2, Fig. 1C), while this was not observed in seedlings inoculated with the Iactivating race 1 Fol004 $\triangle$ Six 5 strain. Notwithstanding this difference, susceptibility to Fol029 was equally reduced following either I or I-2 activation upon co-inoculation as DI and FW were not significantly different between these bioassays.

Co-inoculation of Fol004, which carries Avr1 and Avr2/Six 5, with Fol029 allowed us to check whether cross protection induced by I and $\mathrm{I}-2$ is additive and confers stronger protection than that induced by a single R protein. Comparing FW and DI of Fol004:Fol029 coinoculated plants (Fol004 activates I and I-2) with plants coinoculated with Fol029 and Fol004 $\Delta$ Six 5 (activate solely I) or with Fol004 $\triangle A v r 1$ or Fol007 (both only activate I-2), showed that simultaneous activation of both I and I-2 does not result in enhanced disease reduction compared with co-inoculation with a strain that activates a single immune receptor (Fig. 1, compare treatment 004: 029 with 004 Six 5:029, 004 $\Delta A v r 1: 029$, and 007:029-). The nonadditive nature of the response could indicate that both receptors alone can trigger the maximal cross protection response. Alternatively, if different responses are triggered, they are apparently not additive.

To test whether the endophyte Fo47 confers EMR via a defense mechanism similar as used in cross protection, the extent of disease reduction in tomato plants co-inoculated with Fo47 or an avirulent F. oxysporum f. sp. lycopersici strain with the Fol029 isolate was compared. The FW of Fo47:Fol029 co-inoculated plants was significantly higher than of plants inoculated solely with Fol029 (Fig. 1B), and not different from the mock as mentioned before. In correspondence, the DI of the co-inoculated plants was lower than that of Fol029-inoculated tomato (Fig. 1C). These data confirm that Fo47-based EMR also reduces susceptibility to Fol029 in this tomato cultivar. Notably, the FW of plants co-inoculated with Fo47: Fol029 was at least fivefold higher than of plants co-inoculated with any of the avirulent pathogenic strains and Fol029 (Fig. 1B, brown comparison). In correspondence, the DI in less than half of the Fo47: Fol029-co-inoculated tomato reached a DI of 4 , whereas the majority of the plants co-inoculated with the avirulent $F$. oxysporum f. sp. lycopersici strains showed a DI of at least 4 (Fig. 1C, brown

TABLE 1. Fusarium oxysporum strains that were used to (co)inoculate tomato roots

\begin{tabular}{|c|c|c|c|c|c|}
\hline Fungal strain & Tomato pathogen & $\begin{array}{l}\text { F. oxysporum f. sp. } \\
\text { lycopersici race }\end{array}$ & Relevant genotype & $\begin{array}{l}\text { Avirulent in plants containing } \\
\text { the following }\end{array}$ & Reference \\
\hline Fo47 & No & - & - & - & (Alabouvette 1986) \\
\hline Fol004 & Yes & 1 & $\begin{array}{c}\text { Avrl } \\
\text { Avr2/Six } 5\end{array}$ & $I / I-2$ & (Rep et al. 2005) \\
\hline Fol004 $\Delta$ Six 5 & Yes & 1 & $\begin{array}{c}\text { Avr1 } \\
\text { Avr2/- }\end{array}$ & I/- & (Ma et al. 2015) \\
\hline Fol004 $\triangle A v r l$ & Yes & $1^{\mathrm{a}}$ & $\stackrel{-}{A v r 2 / \text { Six } 5}$ & $-/ I-2$ & (Houterman et al. 2008) \\
\hline Fol007 & Yes & 2 & $\stackrel{-}{A v r 2 / S i x 5}$ & $-/ I-2$ & (Rep et al. 2005) \\
\hline Fol029 & Yes & 3 & $A v r 2^{-} / \operatorname{Six} 5$ & - & (Rep et al. 2005) \\
\hline
\end{tabular}

a Deletion of Avr1 converts this race 1 strain into a race 2 isolate.

b Avr2 of race 3 F. oxysporum f. sp. lycopersici harbors a mutation that prevents detection by I- 2 but retains the virulence function. 
A
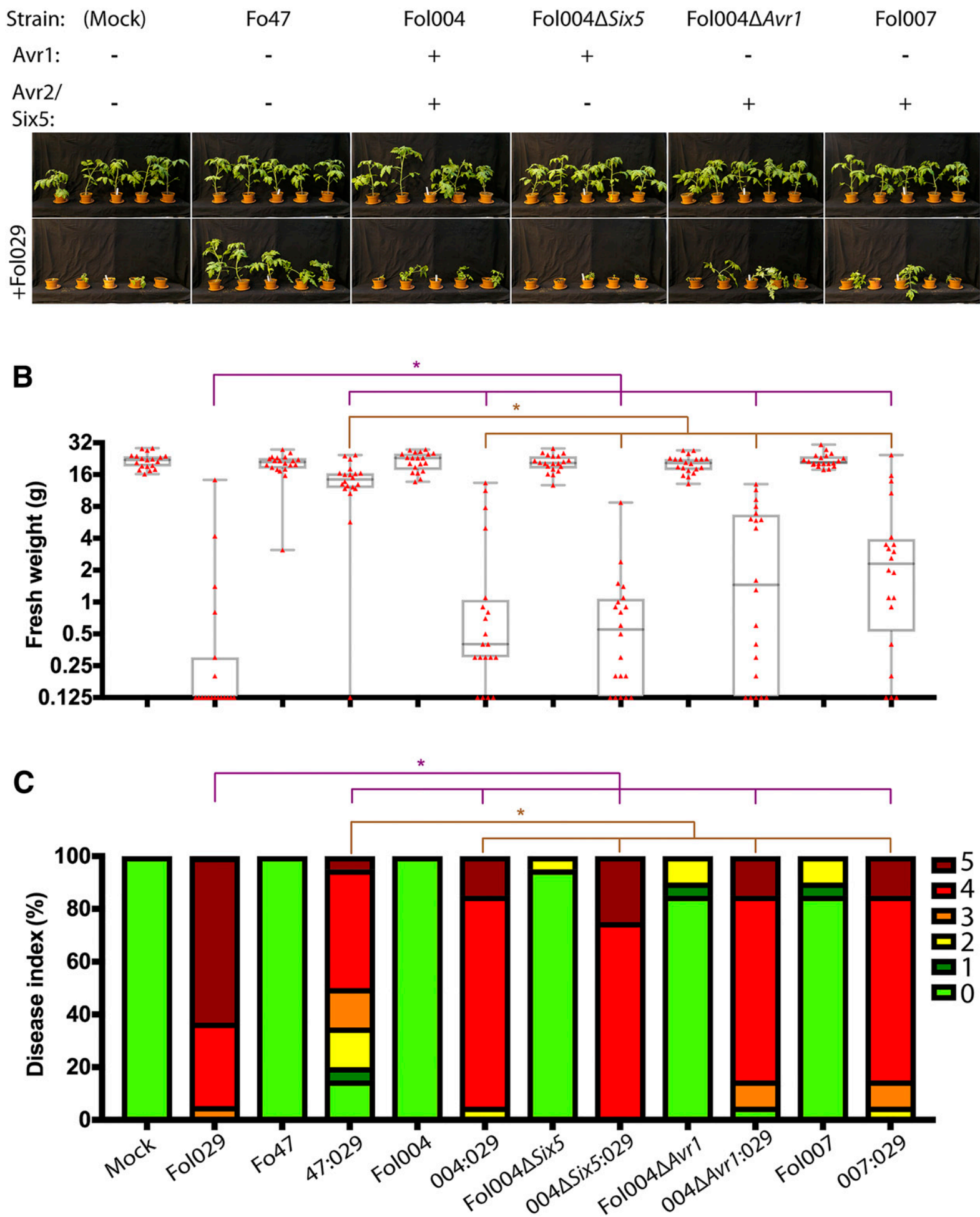

Fig. 1. Co-inoculation of Fo47 with the virulent Fol029 pathogen results in less severe disease symptoms than co-inoculation of Fol029 with avirulent strains. A,

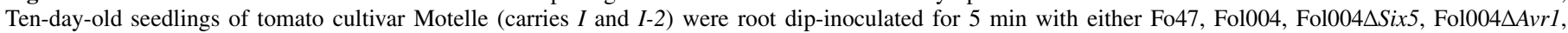
Fol007, and virulent Fol029, or co-inoculated with Fol029 and each of the previously-mentioned strains in a 1:1 ratio using 107 spores/ml (de Lamo et al. 2018; Di et al. 2016). The heading above the photographs (taken 3 weeks postinoculation [wpi]) depicts presence (+) or absence (-) of the cognate Fusarium oxysporum $\mathrm{f}$. sp. lycopersici effectors (Avr1 and Avr2/Six5, respectively) triggering I or I-2, respectively. B, Fresh weight and C, disease index (DI) of inoculated plants were scored 3 wpi. Colors depict the different DIs as indicated in the legend on the right, ranging from 0 (green) being not diseased to 5 (aubergine) plants being dead (de Lamo et al. 2018). The experiment was repeated twice using 20 plants per inoculation and similar results were obtained. Plants were grown in soil within a climate-controlled greenhouse at $25^{\circ} \mathrm{C}$ under a relative humidity of $65 \%$ and with a photoperiod of $16 \mathrm{~h}$. Microconidial spores were isolated from a 5-day-old shake-incubated $(150 \mathrm{rpm})$ culture grown in the dark at room temperature in minimal medium (de Lamo et al. 2018; Di et al. 2016). Data were analyzed using a Mann-Whitney test where * indicates $P$ value $<0.05$ using PRISM 7.0 (GraphPad). The purple line shows single comparisons of every co-inoculated treatment versus solely Fol029 treatment, while the brown line compares Fo47:Fol029 co-inoculation with every single co-inoculated treatment where avirulent $F$. oxysporum f. sp. lycopersici strains were employed. 
comparison). Taken together, both Fo47-based EMR and cross protection reduce wilt disease symptoms. The reduction of disease symptoms upon co-inoculation with the pathogenic Fol029 strain, however, was much more prominent for the Fo47 endophyte than for the avirulent $F$. oxysporum $\mathrm{f}$. sp. lycopersici isolates.

Based on previous studies, an overall higher level of cross protection was anticipated than the one observed in the current study (Fig. 1). For instance, avirulent $F$. oxysporum f. sp. niveum reduces watermelon susceptibility to virulent $F$. oxysporum f. sp. niveum (Biles and Martyn 1989) by at least twofold while coinoculations of tomato with avirulent and virulent $F$. oxysporum $\mathrm{f}$. sp. lycopersici reduces symptom development by $30 \%$ (HuertasGonzalez et al. 1999). In the latter case, even co-inoculation ratios $0.1: 1$ of avirulent/virulent $F$. oxysporum $\mathrm{f}$. sp. lycopersici resulted in a significant disease reduction. We had therefore expected avirulent F. oxysporum f. sp. lycopersici strains to trigger a stronger reduction of susceptibility than the endophyte, as they not only trigger ETI, but possibly also EMR following root colonization. However, since cross protection is much weaker than EMR, either EMR is not triggered by avirulent strains or it is to a much lower extent not able to significantly contribute to resistance.

One possible explanation for the poor protection conferred by avirulent $F$. oxysporum f. sp. lycopersici pathogens might be their ability to compromise (parts of) the tomato immune system using their host-specific effectors (Di et al. 2016, 2017; Gawehns et al. 2014; van Dam et al. 2016). Absence of these effectors in Fo47 explains its inability to cause disease in tomato (van Dam et al. 2016), as transfer of the $F$. oxysporum f. sp. lycopersici pathogenicity chromosome to Fo47 can turn it into a pathogen (Ma et al. 2010; van Dam et al. 2017). The observation that stem colonization by Fo47 is increased in the presence of $F$. oxysporum $\mathrm{f}$. sp. lycopersici (Constantin et al. 2020) supports the idea that, unlike Fo47, avirulent pathogens compromise immunity notwithstanding their inability to cause disease. Indeed, pathogenic $F$. oxysporum strains suppress early host responses like reactive oxygen species accumulation and host cell death more effectively than Fo47 (de Lamo and Takken 2020; Humbert et al. 2015; Olivain et al. 2003). In summary, compared with the endophyte Fo47, avirulent pathogens only weakly reduce susceptibility to $F$. oxysporum f. sp. lycopersici. A possible explanation for this observation is that the Fo47 endophyte is less, or even unable to suppress host immune responses. Presumably, once immune responses are triggered by an endophyte they cannot be suppressed by the effectors present in a virulent strain, or it is to a much lower extent not able to significantly contribute to resistance.

\section{ACKNOWLEDGMENTS}

We thank Ben Cornelissen for critically reading the manuscript and Harold Lemereis and Ludek Tikovsky for plant care.

\section{LITERATURE CITED}

Alabouvette, C. 1986. Fusarium wilt suppressive soils from the Châteaurenard region: Review of a 10-year study. Agronomie 6:273-284.

Bao, J., Fravel, D., Lazarovits, G., Chellemi, D., van Berkum, P., and O’Neill, N. 2004. Biocontrol genotypes of Fusarium oxysporum from tomato fields in Florida. Phytoparasitica 32:9-20.

Biles, C. L., and Martyn, R. D. 1989. Local and systemic resistance induced in watermelons by formae speciales of Fusarium oxysporum. Phytopathology 79:856-860.

Cao, L. X., Blekemolen, M. C., Tintor, N., Cornelissen, B. J. C., and Takken, F. L. W. 2018. The Fusarium oxysporum Avr2-Six5 effector pair alters plasmodesmatal exclusion selectivity to facilitate cell-to-cell movement of Avr2. Mol. Plant 11:691-705.

Catanzariti, A. M., Do, H. T. T., Bru, P., de Sain, M., Thatcher, L. F., Rep, M., and Jones, D. A. 2017. The tomato gene for Fusarium wilt resistance encodes an atypical leucine-rich repeat receptor-like protein whose function is nevertheless dependent on SOBIR1 and SERK3/BAK1. Plant J. 89: 1195-1209.
Constantin, M. E., de Lamo, F. J., Vlieger, B. V., Rept, M., and Takken, F. L. W. 2019. Endophyte-mediated resistance in tomato to Fusarium oxysporum is independent of ET, JA, and SA. Front. Plant Sci. 10:979.

Constantin, M. E., Vlieger, B. V., Takken, F. L. W., and Rep, M. 2020. Diminished pathogen and enhanced endophyte colonization upon coinoculation of endophytic and pathogenic Fusarium strains. Microorganisms 8:544.

de Lamo, F. J., Constantin, M. E., Fresno, D. H., Boeren, S., Rep, M., and Takken, F. L. W. 2018. Xylem sap proteomics reveals distinct differences between $R$ gene- and endophyte-mediated resistance against Fusarium wilt disease in tomato. Front. Microbiol. 9:2977.

de Lamo, F. J., and Takken, F. L. W. 2020. Biocontrol by Fusarium oxysporum using endophyte-mediated resistance. Front. Plant Sci. 11:37.

Dean, R., Van Kan, J. A. L., Pretorius, Z. A., Hammond-Kosack, K. E., Di Pietro, A., Spanu, P. D., Rudd, J. J., Dickman, M., Kahmann, R., Ellis, J., and Foster, G. D. 2012. The top 10 fungal pathogens in molecular plant pathology. Mol. Plant Pathol. 13:414-430.

Di, X., Cao, L., Hughes, R. K., Tintor, N., Banfield, M. J., and Takken, F. L. W. 2017. Structure-function analysis of the Fusarium oxysporum Avr2 effector allows uncoupling of its immune-suppressing activity from recognition. New Phytol. 216:897-914.

Di, X., Gomila, J., Ma, L., van den Burg, H. A., and Takken, F. L. W. 2016. Uptake of the Fusarium effector Avr2 by tomato is not a cell autonomous event. Front. Plant Sci. 7:1915.

Gawehns, F., Houterman, P. M., Ichou, F. A., Michielse, C. B., Hijdra, M., Cornelissen, B. J. C., Rep, M., and Takken, F. L. W. 2014. The Fusarium oxysporum effector Six6 contributes to virulence and suppresses I-2mediated cell death. Mol. Plant-Microbe Interact. 27:336-348.

Houterman, P. M., Cornelissen, B. J., and Rep, M. 2008. Suppression of plant resistance gene-based immunity by a fungal effector. PLoS Pathog 4: e1000061.

Houterman, P. M., Ma, L., van Ooijen, G., de Vroomen, M. J., Cornelissen, B. J. C., Takken, F. L. W., and Rep, M. 2009. The effector protein Avr2 of the xylem-colonizing fungus Fusarium oxysporum activates the tomato resistance protein I-2 intracellularly. Plant J. 58:970-978.

Huertas-Gonzalez, M. D., Ruiz-Roldan, M. C., Di Pietro, A., and Roncero, M. I. G. 1999. Cross protection provides evidence for race-specific avirulence factors in Fusarium oxysporum. Physiol. Mol. Plant Pathol. 54: 63-72.

Humbert, C., Aimé, S., Alabouvette, C., Steinberg, C., and Olivain, C. 2015. Remodelling of actin cytoskeleton in tomato cells in response to inoculation with a biocontrol strain of Fusarium oxysporum in comparison to a pathogenic strain. Plant Pathol. 64:1366-1374.

Laterrot, H. S. 1993. Revised list of near isogenic tomato lines in Moneymaker type with different genes for disease resistances. Rep. Tomato Genet. Coop. 43:79-80.

Ma, L. J., van der Does, H. C., Borkovich, K. A., Coleman, J. J., Daboussi, M. J., Di Pietro, A., Dufresne, M., Freitag, M., Grabherr, M., Henrissat, B., Houterman, P. M., Kang, S., Shim, W. B., Woloshuk, C., Xie, X., Xu, J. R., Antoniw, J., Baker, S. E., Bluhm, B. H., Breakspear, A., Brown, D. W., Butchko, R. A., Chapman, S., Coulson, R., Coutinho, P. M., Danchin, E. G., Diener, A., Gale, L. R., Gardiner, D. M., Goff, S., Hammond-Kosack, K. E., Hilburn, K., Hua-Van, A., Jonkers, W., Kazan, K., Kodira, C. D., Koehrsen, M., Kumar, L., Lee, Y. H., Li, L., Manners, J. M., Miranda-Saavedra, D., Mukherjee, M., Park, G., Park, J., Park, S. Y., Proctor, R. H., Regev, A., Ruiz-Roldan, M. C., Sain, D., Sakthikumar, S., Sykes, S., Schwartz, D. C., Turgeon, B. G., Wapinski, I., Yoder, O., Young, S., Zeng, Q., Zhou, S., Galagan, J., Cuomo, C. A., Kistler, H. C., and Rep, M. 2010. Comparative genomics reveals mobile pathogenicity chromosomes in Fusarium. Nature 464:367-373.

Ma, L. S., Houterman, P. M., Gawehns, F., Cao, L. X., Sillo, F., Richter, H., Clavijo-Ortiz, M. J., Schmidt, S. M., Boeren, S., Vervoort, J., Cornelissen, B. J. C., Rep, M., and Takken, F. L. W. 2015. The AVR2-SIX5 gene pair is required to activate $I$-2-mediated immunity in tomato. New Phytol. 208: 507-518.

Michielse, C. B., and Rep, M. 2009. Pathogen profile update: Fusarium oxysporum. Mol. Plant Pathol. 10:311-324.

Olivain, C., Trouvelot, S., Binet, M. N., Cordier, C., Pugin, A., and Alabouvette, C. 2003. Colonization of flax roots and early physiological responses of flax cells inoculated with pathogenic and nonpathogenic strains of Fusarium oxysporum. Appl. Environ. Microbiol. 69:5453-5462.

Pieterse, C. M. J., Zamioudis, C., Berendsen, R. L., Weller, D. M., Van Wees, S. C. M., and Bakker, P. A. H. M. 2014. Induced systemic resistance by beneficial microbes. Annu. Rev. Phytopathol. 52:347-375.

Rep, M., Meijer, M., Houterman, P. M., van der Does, H. C., and Cornelissen, B. J. C. 2005. Fusarium oxysporum evades I-3-mediated resistance without altering the matching avirulence gene. Mol. Plant-Microbe Interact. 18: $15-23$.

Simons, G., Groenendijk, J., Wijbrandi, J., Reijans, M., Groenen, J., Diergaarde, P., Van der Lee, T., Bleeker, M., Onstenk, J., de Both, M., 
Haring, M., Mes, J., Cornelissen, B. J. C., Zabeau, M., and Vosa, P. 1998. Dissection of the Fusarium I2 gene cluster in tomato reveals Six homologs and one active gene copy. Plant Cell 10:1055-1068.

Takken, F. L. W., and Rep, M. 2010. The arms race between tomato and Fusarium oxysporum. Mol. Plant Pathol. 11:309-314.

Tamietti, G., Ferraris, L., Matta, A., and Gentile, I. A. 1993. Physiological responses of tomato plants grown in Fusarium suppressive soil. J. Phytopathol. 138:66-76

van Dam, P., Fokkens, L., Ayukawa, Y., van der Gragt, M., ter Horst, A., Brankovics, B., Houterman, P. M., Arie, T., and Rep, M. 2017. A mobile pathogenicity chromosome in Fusarium oxysporum for infection of multiple cucurbit species. Sci. Rep. 7:9042.

van Dam, P., Fokkens, L., Schmidt, S. M., Linmans, J. H. J., Kistler, H. C., Ma, L. J., and Rep, M. 2016. Effector profiles distinguish formae speciales of Fusarium oxysporum. Environ. Microbiol. 18:4087-4102.

van der Does, H. C., Constantin, M. E., Houterman, P. M., Takken, F. L. W., Cornelissen, B. J. C., Haring, M. A., van den Burg, H. A., and Rep, M. 2018. Fusarium oxysporum colonizes the stem of resistant tomato plants, the extent varying with the $R$-gene present. Eur. J. Plant Pathol. 154: $55-65$. 